\title{
ESTADO NUTRICIONAL Y ANEMIA FERROPÉNICA EN GESTANTES ADOLESCENTES DEL CENTRO DE SALUD ALTO DE LA ALIANZA
}

\author{
NUTRITIONAL STATUS ANDIRON DEFICIENCY ANEMIA INPREGNANT \\ TEEN HEALTH CENTER ALTO DE LA ALIANZA
}

\author{
Soledad A. Bornás Acosta'; Vicente F. Chambilla Quispe
}

\begin{abstract}
RESUMEN
El propósito de la presente investigación es relacionar la anemia con el nivel nutricional de las gestantes adolescentes que acudieron al Centro de Salud Alto de la Alianza durante el periodo de 2012, en el departamento de Tacna. La muestra se seleccionó en adolescentes gestantes comprendidas entre las edades de 12 a 19 años que visitaron en el primer, segundo y tercer trimestre de gestación. Se ha utilizado como instrumento de recolección de datos las fichas e historias clínicas, toma de muestras de sangre para el análisis de hemoglobina y una encuesta para saber el conocimiento del nivel nutricional. En conclusión del total de 25 adolescentes gestantes, el 52\% presenta anemia, siendo el tipo leve el más predominante. Además, se ha determinando que el conocimiento nutricional que poseen es inadecuado.
\end{abstract}

Palabras clave: Anemia, nivel nutricional, gestantes adolescentes, hemoglobina.

\section{ABSTRACT}

The purpose of this research is to relate the nutritional anemia of pregnant adolescents who attended the Health Center Alto de la Alianza during the period of 2012, in the department of Tacna. The sample was selected in pregnant teenagers between the ages of 12 to 19 years who visited in the first, second and third trimester. It has been used as an instrument for data collection sheets and medical records, blood sampling for analysis of hemoglobin and a survey to know the nutritional knowledge. In conclusion of the 25 pregnant teenagers, $52 \%$ have anemia, mild type being the most predominant. Furthermore, it has been determined that they have nutritional knowledge is inadequate

Keywords: anemia, level of nutritional, pregnant teenagers, hemoglobin.

\section{INTRODUCCIÓN}

En Latinoamérica, las tasas globales de fecundidad, mortalidad infantil y mortalidad materna son elevadas. Asimismo, la prevalencia de malnutrición infantil es alta y a menudo se traduce en retraso del crecimiento y del desarrollo y en enfermedades de origen alimentario, tales como la anemia ferropénica. En el Perú, la población muestra un crecimiento acelerado y la tasa de mortalidad materno infantil es alta. La situación representada por estos indicadores se torna más patente y alarmante cuando el análisis se estratifica por zonas geográficas [5]. Según los resultados de la Encuesta Demográfica y de Salud Familiar, la tasa global de fecundidad es más alta en la selva que en otras zonas del país, al igual que la proporción de adolescentes embarazadas y la mortalidad materno infantil $[2],[4]$.

Entre los problemas clínicos destaca la anemia, cuya prevalencia es especialmente elevada en los países en desarrollo oscila entre 20 y $40 \%$ en mujeres no gestantes.

Se ha observado que la causa de la enfermedad, en dichos países es la deficiencia de hierro y constituye un problema de salud muy difundido, especialmente en mujeres embarazadas. No obstante, las actividades de los programas nacionales de control son insuficientes y se han limitado a incluir suplementos de hierro en la dieta

\footnotetext{
Bióloga, Magíster en Ciencias en Gestión Ambiental y Desarrollo Sostenible. Facultad de Ciencias de la UNJBG.

${ }^{2}$ Biólogo, Magíster en Salud Pública. Facultad de Ciencias de la UNJBG.
} 
de las embarazadas que acuden a los servicios de control prenatal. Por otra parte, la anemia es la complicación más frecuente del embarazo y está asociada con tasas elevadas de parto pretérmino, bajo peso al nacer y mortalidad perinatal [3], [5]. Se considera anemia cuando las cifras de hemoglobina se encuentran por debajo del 11,0 g/L de sangre y un hematocrito del $33 \%$ o menos, durante el tercer trimestre de la gestación [4], [6].

En el Perú, la anemia se ha estudiado en poblaciones situadas principalmente en la costa.

En los zonas alejadas de Tacna se vienen realizando, periódicamente, descartes y promoción de salud en este tema; sin embargo, las creencias de la población, muy arraigadas, los llevan a ignorar, muchas veces.

Las recomendaciones acerca del cuidado que debe tener una gestante son la buena alimentación y el consumo de los suplementos férricos y de ácido fólico, que son repartidos gratuitamente por el Ministerio de Salud (MINSA). Pero nos hace pensar en la posibilidad de que haya más del $50 \%$ de gestantes anémicas, en las que están comprendidas las gestantes adolescentes [8].

\section{OBJETIVO}

Fue determinar el estado nutricional y la anemia ferropénica de las gestantes adolescentes del Centro de Salud Alto de la Alianza durante el año 2012.

\section{MATERIALES Y MÉTODOS}

\subsection{Descripción}

El estudio es de tipo prospectivo, descriptivo y transversal, tomando como muestra a un determinado número de gestantes adolescentes que acuden a sus primeros controles prenatales en el Centro de Salud Alto de la Alianza, del distrito del mismo nombre.

Las muestras obtenidas fueron procesadas en el Laboratorio de Bioquímica y Nutrición de la Facultad de Ciencias de la UNJBG.

\subsection{Metodología}

El método de muestreo para el presente estudio es no probabilístico consecutivo. Se toma en cuenta los criterios de inclusión, es decir, que sólo se considera a gestantes adolescentes que acuden al Centro de Salud Alto de la Alianza, cuyas edades se encuentran entre los 12 y 19 años.
La recopilación de los datos se realizó mediante revisión de historias clínicas con el apoyo del personal asistencial del área de obstetricia.

En la determinación de anemia ferropénica, hemos tomado una muestra de sangre de cada paciente para evaluar el nivel de hemoglobina en sangre, procesándolo en el laboratorio de Bioquímica y Nutrición de la Facultad de Ciencias, ubicado en la Ciudad Universitaria de la UNJBG.

Además se evaluó el nivel nutricional tanto de conocimiento como aptitud hacia su buen consumo, propicios para su estado gestacional. La evaluación se realizó mediante una pequeña encuesta de nutrición.

Para la determinación de anemia, se procedió a extraer una muestra de sangre venosa usando un ligador, agujas descartables, alcohol y algodón para desinfectar la zona de punción.

Con la muestra de sangre obtenida se determinó el nivel de hierro sérico (hemoglobina), utilizando un método colorimétrico sin desproteinizador (Wiener lab.) y con el espectrofotómetro de 540nm de longitud de Onda.

También se determinó las medidas antropométricas y, con los datos de una encuesta, se estableció el nivel nutricional de las gestantes que acudieron a dicho Centro de Salud durante el año 2012.

\section{RESULTADOS}

Los resultados fueron ordenados mediante tablas, utilizando programa Microsoft Excel 2007, y teniendo en cuenta la edad de las gestantes adolescentes (de 12 a 19 años) y el lugar de procendencia (Centro de Salud Alto de la Alianza, ubicado en el distrito del mismo nombre).

El total de pacientes adolescentes evaluadas durante el tiempo de embarazo, en el año 2012, fueron 25 .

A continuación se presenta en la Tabla $\mathrm{N}^{\circ} \mathrm{O} 1 \mathrm{el}$ total de adolescentes que acudieron al control de embarazo, el año 2012, donde el 8\% de las gestantes estaban en la última etapa de embarazo, el $28 \%$ se encontraba en la primera etapa y el $64 \%$ de las gestantes, en el segundo trimestre de gestación.

Asimismo, se puede observar los niveles de hemoglobina que se encontraron entre los $8,5 \mathrm{~g} / \%$, el más bajo, y $14 \mathrm{~g}$ /\% el más elevado. 
Tabla $\mathrm{N}^{\circ}$ 01: Adolescentes gestantes que acudieron al control de embarazo durante el año 2012.

\begin{tabular}{|c|c|c|c|}
\hline No. & EDAD & $\begin{array}{l}\text { HEMOGLOBINA } \\
\text { (HB: } \mathrm{g} / \%)\end{array}$ & $\begin{array}{l}\text { TRIMESTRE } \\
\text { (CONTROL) }\end{array}$ \\
\hline 01 & 19 & 10,2 & III \\
\hline 02 & 15 & 8,5 & II \\
\hline 03 & 18 & 11,2 & III \\
\hline 04 & 16 & 11,3 & I \\
\hline 05 & 16 & 10,5 & II \\
\hline 06 & 16 & 10,0 & II \\
\hline 07 & 19 & 8,6 & II \\
\hline 08 & 18 & 10,5 & II \\
\hline 09 & 18 & 11,4 & II \\
\hline 10 & 18 & 9,9 & II \\
\hline 11 & 15 & 10,0 & $\mathrm{I}$ \\
\hline 12 & 19 & 11,5 & II \\
\hline 13 & 18 & 12,0 & II \\
\hline 14 & 18 & 10,9 & II \\
\hline 15 & 16 & 12,0 & $\mathrm{I}$ \\
\hline 16 & 19 & 11,2 & II \\
\hline 17 & 19 & 14,0 & $\mathrm{I}$ \\
\hline 18 & 16 & 12,0 & I \\
\hline 19 & 17 & 11,2 & II \\
\hline 20 & 17 & 12,7 & II \\
\hline 21 & 16 & 13,0 & II \\
\hline 22 & 18 & 10,1 & II \\
\hline 23 & 19 & 10,8 & I \\
\hline 24 & 18 & 10,4 & II \\
\hline 25 & 18 & 9,5 & I \\
\hline
\end{tabular}

Fuente: Propia

En la Tabla $\mathrm{N}^{\circ} \mathrm{O} 2$ y Gráfico $\mathrm{N}^{\circ} \mathrm{O} 1$ se muestra que el $52 \%$ de las gestantes adolescentes se encontraban anémicas y el $48 \%$ presentaban un nivel de hemoglobina normal.

Tabla $\mathbf{N}^{\mathbf{o}}$ 02: Porcentaje de adolescentes gestantes que presentan anemia.

\begin{tabular}{|c|c|c|c|c|}
\hline \multicolumn{2}{|c|}{ CON ANEMIA } & \multicolumn{2}{|c|}{ SIN ANEMIA } & \multirow{2}{*}{ TOTAL } \\
\hline No. & $\%$ & No. & $\%$ & \\
\hline 13 & 52 & 12 & 48 & 25 \\
\hline
\end{tabular}

Fuente: Propia

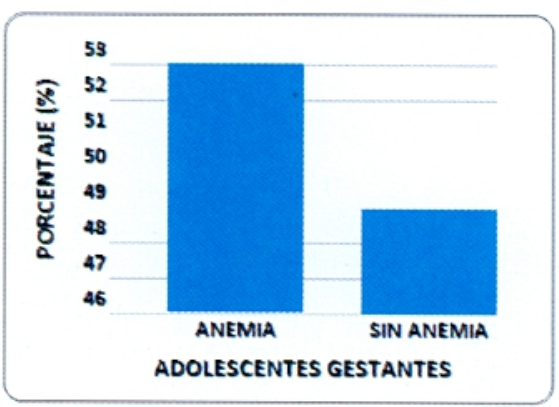

Gráfico $\mathrm{N}^{\circ} 01$ : Porcentaje de adolescentes gestantes con anemia.

En la Tabla $\quad \mathrm{N}^{\circ} 03$ y Gráfico $\mathrm{N}^{\circ} \mathrm{O} 2$ se muestra los tipos de anemia relacionadas con la edad de las adolescentes gestantes, donde el $44 \%$ muestra anemia leve y tan sólo el $8 \%$, anemia moderada.
Tabla $\mathrm{N}^{\circ}$ 03: Tipos de anemia relacionados con la edad de las adolescentes gestantes.

\begin{tabular}{|c|c|c|c|c|c|}
\hline \multirow{2}{*}{ EDAD } & \multicolumn{3}{|c|}{ ANEMIA } & SIN & \multirow{2}{*}{ TOTAL } \\
\cline { 2 - 5 } & LEVE & MODERADA & GRAVE & ANEMIA & \\
\cline { 2 - 5 } & $\%$ & $\%$ & $\%$ & $\%$ & $\%$ \\
\hline $10-15$ & 4 & 4 & 00 & 48 & 100 \\
\hline $16-19$ & 40 & 4 & 00 & 0 & 100 \\
\hline TOTAL & 44 & 8 & 00 & 48 & 100 \\
\hline
\end{tabular}

Fuente: Propia

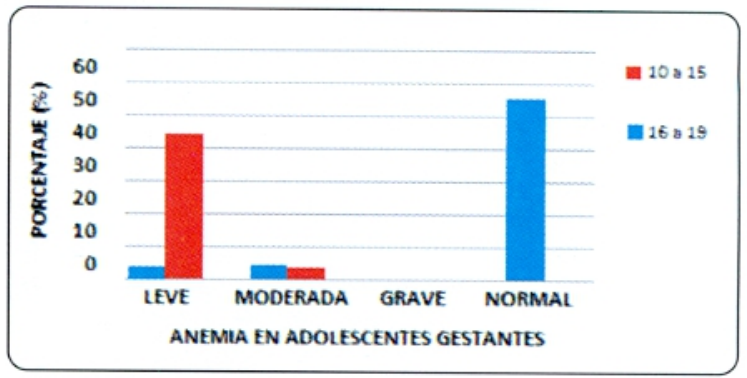

Gráfico $\mathbf{N}^{\mathbf{o}}$ o2: Tipo de anemia de adolescentes gestantes.

En la Tabla 04 y Gráfico $\mathrm{N}^{\circ} 03$ se muestra la frecuencia de tipos de anemia de las mismas adolescentes embarazadas, donde el 23,08 \% presenta anemia leve en el primer trimestre. En cambio el $63,84 \%$ presentan anemia leve y un $15,38 \%$, anemia moderada, durante el segundo trimestre. Por último, sólo el 7,69 \% tenían anemia leve durante el tercer trimestre de gestación.

Tabla $\mathbf{N}^{\circ}$ 04: Frecuencia de tipos de anemia en adolescentes gestantes, según trimestre gestacional.

\begin{tabular}{|c|c|c|c|c|}
\hline \multirow{2}{*}{ TRIMESTRE } & \multicolumn{3}{|c|}{ ANEMIA } & \multirow{2}{*}{ TOTAL } \\
\cline { 2 - 4 } & LEVE & MODERADA & GRAVE & \multirow{n}{*}{} \\
\cline { 2 - 4 } & $\%$ & $\%$ & $\%$ & \\
\hline I & 23.08 & 00.00 & 00 & 100 \\
\hline II & 63.84 & 15.38 & 00 & 100 \\
\hline III & 7.69 & 00.00 & 00 & 100 \\
\hline
\end{tabular}

Fuente: Propia

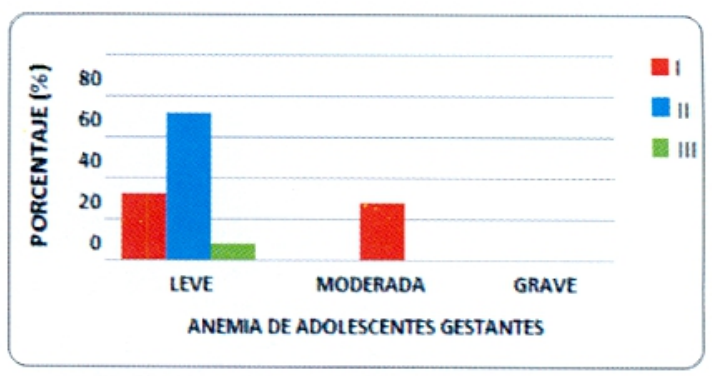

Gráfico $\mathrm{N}^{\mathbf{0}}$ 03: Frecuencia de tipos de anemia en adolescentes embarazadas.

En la Tabla $\mathrm{N}^{\circ} 05$ y Gráfico $\mathrm{N}^{\circ} \mathrm{O} 4$ se muestra la frecuencia de alimentos que fueron consumidos durante el periodo de gestación. 
Tabla $\mathbf{N}^{\mathbf{0}} \mathbf{0 5}$ : Frecuencia de alimentos consumidos por adolescentes embarazadas.

\begin{tabular}{|l|c|c|c|}
\hline \multirow{2}{*}{ NUTRIENTES } & \multicolumn{3}{|c|}{ CONSUMO } \\
\cline { 2 - 4 } & LEVE & MODERADO & ABUNDANTE \\
\cline { 2 - 4 } & $\%$ & $\%$ & $\%$ \\
\hline GLUCIDOS & 5 & 25 & 70 \\
\hline LIPIDOS & 50 & 20 & 30 \\
\hline PROTEINAS & 18 & 80 & 2 \\
\hline VITAMINAS & 85 & 10 & 5 \\
\hline MINERALES & 86 & 9 & 5 \\
\hline AGUA & 5 & 75 & 20 \\
\hline
\end{tabular}

Fuente: Propia

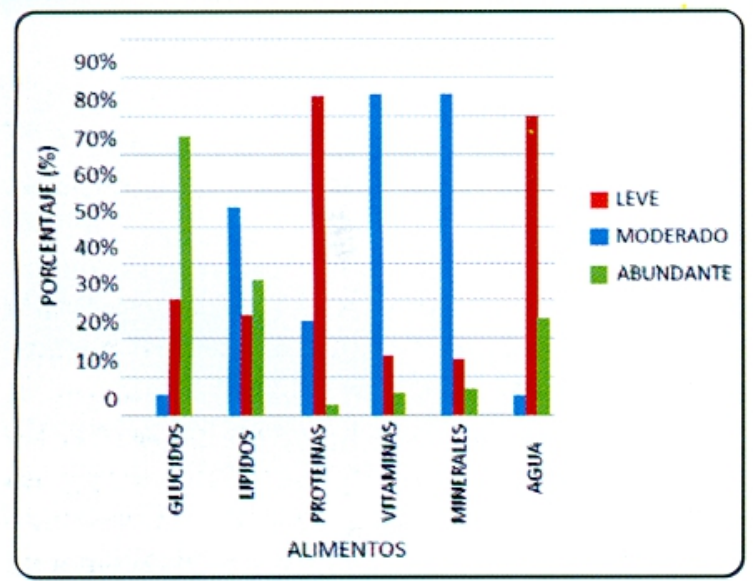

Gráfico $\mathrm{N}^{\mathbf{o}}$ 04: Frecuencia de alimentos consumidos por adolescentes embarazadas

\section{IV.DISCUSIÓN}

En la Tabla $\mathrm{N}^{\circ} \mathrm{O} 2$ se muestra que el $52 \%$ de las gestantes adolescentes presentan anemia y el $48 \%$ no. Estos resultados concuerdan con las investigaciones realizadas por Díaz y Col. (2004) donde manifiestan que la presencia de anemia en las embarazadas adolescentes fue de un $49,36 \%$.

En la Tabla $\mathrm{N}^{\circ} \mathrm{O}$ se aprecia que el tipo de anemia más frecuente en las gestantes adolescentes es leve (44\%). El mayor número oscila entre las edades de 16 a 19 años y en un $40 \%[1]$, seguido del $8 \%$ de adolescentes gestantes con anemia moderada. No se presentan adolescentes con anemia grave.

En la Tabla $\mathrm{N}^{\circ} 04$ podemos apreciar la mayor parte de las adolescentes gestantes presentan anemia. El 69.23 \% realizaron su control en el segundo trimestre gestacional. Se da un elevado índice del tipo de anemia leve. Solamente un 23,08 $\%$ de las gestantes adolescentes hicieron su control en el primer trimestre, presentando un cuadro de anemia leve.

La formación de un nuevo ser determina una serie de exigencias en la madre. Una es la alimentación, la cual debe reunir las sustancias nutritivas que requieren, tanto la madre como el niño por nacer $[9],[11]$.

Según la Tabla $\mathrm{N}^{\circ} 05$, el $70 \%$ de las adolescentes embarazadas consumen carbohidratos en forma abundante; el $80 \%$ de embarazadas consume proteínas moderadamente y que el $85 \%$ consume suplementos vitamínicos y minerales en forma leve. La ingesta de agua es realizada con moderación por el $75 \%$.

La actividad diaria de la mujer (sin estar embarazada) tiene un gasto aproximado de 2.000 calorías. Durante los primeros meses de embarazo, la cantidad de calorías debe mantenerse entre 2.000 y 2.200 por día.

Conforme la gestación progresa los requerimientos del niño y el consumo de calorías por parte de la madre puede llegar a 2.600 . Generalmente la actividad de la madre disminuye. No es necesario aumentar la ingesta calórica [5].

\section{V.- CONCLUSIONES}

- De la población de gestantes adolescentes estudiada, el $52 \%$ presentan anemia de tipo leve.

- Según los datos antropométricos las embarazadas adolescentes aparentemente no presentan desnutrición.

- Las madres adolescentes atendidas en el Centro de Salud Alto de la Alianza se caracterizan por presentar condiciones socioeconómicas y nutricionales desfavorables, condicionando mayor morbilidad materna y morbi-mortalidad neonatal; por lo tanto, en esta zona el embarazo del adolescente se debe considerar como un grupo de alto riesgo materno-perinatal.

\section{REFERENCIAS BIBLIOGRÁFICAS}

[1]ALiagA, M.: "Características Sociales de Adolescentes Embarazadas del Instituto Materno Perinatal". Tesis para optar el grado de Maestro en Salud Comunitaria con mención en Materno Infantil y Población. Universidad Particular Cayetano Heredia. Lima. 1996.

[2]BECERRA, C. y col. Prevalencia de Anemia en gestantes, Hospital Regional de Pucallpa, Perú. Rev. Panam Salud Pública/ Pan.Am.J.PublicHealth 3(5), 1998. 
[3]Boletín- Instituto Nacional de Salud. 2005; 11 (7-8) julio-agosto.

[4]CERVANTES, R.; Watanabe, T.: "La Adolescencia como problema de Salud Reproductiva". XI Congreso Peruano de Obstetricia y Ginecología. Sociedad Peruana de Obstetricia y Ginecología. lima 1994.

[5]CLARK S. Iron Deficiency Anemia. Nutrition in Clinical Practice. 2008. 23; 2: 128-141.

[6]CHÁVEZ, I.: "Embarazo en Adolescentes en el Hospital Regional Cayetano Heredia de Piura". Tesis para Optar el Grado de Maestro en Medicina. Universidad Particular Cayetano Heredia. Linia,. 1989.

[7]GONZÁLEZ, G. "La Adolescencia en el Perú". Universidad Peruana Cayetano Heredia. Lima. 1994.

[8]GUTIÉRREZ, I.:"Factores de Riesgo Asociados al Embarazo en Primíparas Adolescentes en el Hospital Arzobispo Loayza de Lima y el Hospital Nacional de PucalIpa". Tesis para optar el Título de Médico Cirujano. Universidad Particular Cayetano Heredia. Lima. 1993.

[9]Instituto Nacional de Estadística e Informática. "Encuesta Demográfica y de Salud Familiar 1991/1992. Perú". Asociación Benéfica PRISMA. DHS Macro Internacional Ine. Lima. 1992.

[10]Instituto Nacional de Estadística e Informática. "La Salud de la Niñez, Adolescencia y de la Mujer en el Perú". Lima. 1994.

[11]MOLINA, R.; ALARCÓN, G.: Lueng'o, X.; et. al.: "Estudio Prospectivo de factores de Riesgo en Adolescentes Embarazadas". Revista Chilena de Obstetricia y Ginecología. 198 8; 5 3(1): 27-34.

[12]OLINA, R.; Luengo, X.; Guarda, P.; González, E.; Jara, G. "Adolescencia, Sexualidad y Embarazo". Serie Científica Médica 2. Centro de Extensión Biomédica, Facultad de Medicina, Universidad de Chile. 1991.

[13]MONROY, A. "El Embarazo en la Adolescencia: La Experiencia en América Latina". Salud Reproductiva de las
Américas. Organización Panamericana de la Salud. P. 132-146. 1992.

[14]MUNITZ, M; SILBER, T. "El Embarazo en Adolescentes: Enfoque Clínico Epidemiológico". Salud Reproductiva en las Américas. Organización Panamericana de la Salud. P. 96-124.1992.

[15]Organización Panamericana de la Salud. "La Salud de los Adolescentes y los Jóvenes en las Américas: Escribiendo el Futuro". Comunicación para la Salud N'6. Washington D.C. E.U.A. 1995.

[16]PARRA, J.PINEDO, A. TÁVARA, L. VEREA U, D. A YA S T A, C. "Comportamiento Reproductivo de las Adolescentes. Hospital de Apoyo María Auxiliadora de Lima". X Congreso Peruano de Obstetricia y Ginecología. Perú. Setiembre 1990.

[17]ROBINOVICH, J.; PINERO, L),; FERMELLY, K.; BEARD, J. "Efectos de la Edad Materna en el Estado de Salud del Neonato". Revista Chilena de Obstetricia y Ginecología. 1994; 54(4): 293-300.

[18]ROMERO, M.: Maddaleno, M.; Munist, M. "Salud Reproductiva". Manual de Medicina de la Adolescencia. Organización Panamericana de la Salud. Serie Paltex para ejecutores de programas $\mathrm{N}^{\prime}$ 20. P.4 73501.1992 .

[19]RUBARTH, G. "La Adolescente Embarazada". Grupo Editor Latinoamericano. Argentina. 1994.

[20]SERRANO, C. "La Salud Integral de los Adolescentes y los Jóvenes, su Promoción y su Cuidado". La Salud del Adolescente y el Joven. Organización Panamericana de la Salud. Pub. Cient. N' 552. Washington. EUA. 1995.

[2 1]SILBER, T. "El embarazo en la Adolescencia". La Salud del Adolescente y el Joven. Organización Panamericana de la Salud. Pub. Cient. N' 552. Washington. EUA. 1995.

[22]The pathfinder fund and population council. "La Actividad Sexual y la Maternidad entre las Adolescentes en América Latina y el Caribe: Riesgos y consecuencias. 1990. 
〔23〕TICONA RENDÓN, MANUEL: "El Recién Nacido. Morbi-Mortalidad". Universidad Nacional Jorge Basadre G. de Tacna. Perú. 1995.

[24]VIEGAS D. Nutrición durante el embarazo. En: Zighelboim I, Guariglia D, editores. Clínica Obstétrica. Caracas: Editorial Disinlimed; 2001.p.147-154.

[25]VIEL, B. "El Embarazo de Adolescencia, Problema de Salud Pública". Boletín Asociación de Protección de la Familia. p, 1520. Santiago de Chile. 1986.
[26]VITE, F. Incidencia de anemia ferropénica y factores asociados en las gestantes del distrito de Rapayan, Ancash, Perú: Periodo mayo 2010 - marzo 2011. Médico Cirujano. Universidad Científica del Sur, Lima, Perú.

\section{Correspondencia:}

Soledad A. Bornás Acosta

Ciudad Universitaria fundo "Los Granados" Av. Miraflores s/n Tacna - Perú

Vicente Freddy Chambilla Quispe

Ciudad Universitaria fundo "Los Granados"

Av. Miraflores s/n Tacna - Perú

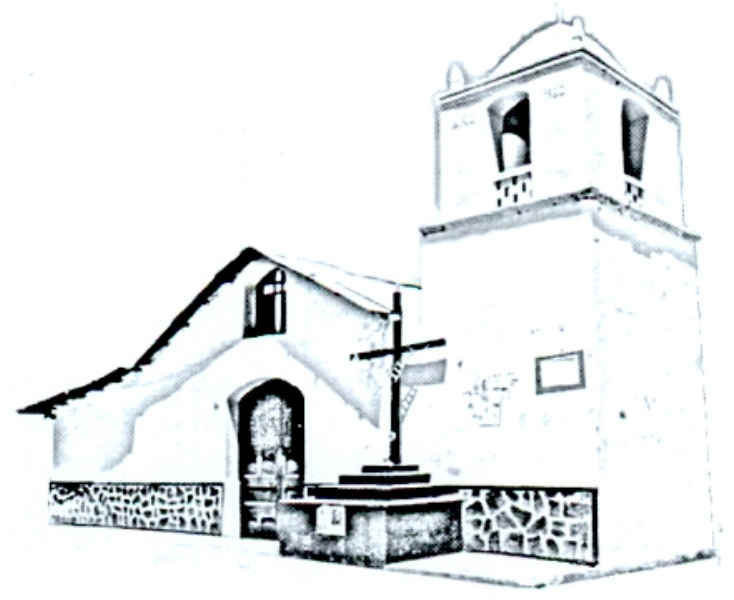

Iglesia San Isidro de Yacango, Torata 